\title{
PCR Detects the DNA Vaccines in mice Vaccinated via and Construction Containing Hsp70 and GP5 /M protein of PRRSV
}

\author{
ZUO Lan ${ }^{1}$,YAN Qi-gui ${ }^{1,2^{*}}$, CHEN Shi-jie ${ }^{3}$, LEI Yan ${ }^{1}$,WANG Xu ${ }^{1}$, \\ (1.Animal Biotechnology Center, Sichuan Agricultural University, Ya'an, Sichuan, 625014,China ;2.Key \\ laboratory of Animal Disease and Human Health of Sichuan Province, Sichuan Agricultural \\ University,Ya'an,625014,China;3.Sichuan Entry-exit Inspection and Quarantine Bureau,Chengdu,610041,China)
}

$\begin{array}{lcrr}\text { Abstract-Construction } & \text { of } & \text { Eukaryotic } & \text { recombinant } \\ \text { expression } & \text { Vector } & \text { pCI-ORF5-ORF6/ }\end{array}$ pCI-ORF5-ORF6B-Hsp70 Containing Hsp70 and PRRSV GP5/M (encoded by ORF5 and ORF6 genes), and to study its immune effect. After being identified by enzyme analysis and nucleotide sequencing test, the repression vector plasmid was transfected into COS-7 cells. The transient expression protein was detected by Western-blotting. The immunogenicities of this DNA vaccine constructs were firstly investigated in a mouse moder. IFN- $\gamma$, IL-4 of cytokine, and the spleen T-lymphocyte subgroup quantity (CD4+/CD8+) were detected, DNA vaccine distribution in mice by PCR.The result shows that the recombinant plasmid pCI-ORF5-ORF6-Hsp70 could induce higher response of cellular immune responses and specific immune responses in mouse, the DNA Vaccines in mice Vaccinated via as heart and liver, lung and kidney, muscle and brain each time step after immunity. providing the clinical basic data and theoretical basis for success of the DNA vaccine development.

Index Terms-PRRSV; DNA vaccine; Hsp70; DNA immune; distribute

\section{INTRODUCTION}

Porcine reproductive and respiratory syndrome (PRRS) is induced by porcine reproductive and respiratory syndrome virus (PRRSV) and characterized with reproductive failure in pregnant sows, reproductive abnormalities including still birth, fetal mummies, weak piglets, and respiratory disease in young pigs[1].Two commercial vaccines currently used to prevent PRRS are attenuated vaccine and inactivated vaccine, both of which suffer from poor immune protective effect and danger of toxic exposures. It is a hotpot of the research to develop new type gene engineering vaccines. PRRS gene engineering vaccine is safe, manually modifiable, easy to prepare and able to induce in cellular immunity and humoral immunity synchronously, showing great potential for prevention and control of this disease[2]. The known PRRSV structural proteins which have neutralization epitope are GP4(encoded by ORF4), E(encoded by ORF5), M(encoded by ORF6). E proteins have the ability to induce neutralizing antibody, while $\mathrm{M}$ proteins can induce specific cellular immunity. Using PRRSV modified GP5 and M protein genes as candidate genes, Jiang Yunbo constructed double gene co-expressed eukaryotic expression plasmid and detected rather high specific cellular immune response in immuned mice and weaned piglets[3]. The eukaryotic expression plasmid PRRSV-ORF5-ORF6 constructed by Rossw can activate the cell-mediated and antigen specific T-cell immune response[4]. These studies proved that GP5-M gene co-expression can be used as an important candidate gene for PRRS DNA vaccine[5]. The human heat shock protein 70 (Hsp70) is an immunodominant antigen, which can induce specific Th1 cell reaction and participate in immune response[6]. By activating the chimerism of genes with adjuvant effect in one eukaryotic expression plasmid, protective immunity is enhanced, while toxic side effects are reduced. Now, the research of $\mathrm{Hsp} 70$ is progressing. Human mycobacterium tuberculosis Tubercle Bacillus (TB) Hsp70 is used to enhance the immune effect of PRRSV gene vaccine in this study. Uing pCI as target gene, PRRSV ORF5, ORF6 as aim gene, eukaryotic expression plasmid containing Hsp70, PRRSV ORF5, and ORF6 was constructed and immune tested in mice. Results show that Hsp70 can enhance immune function of PRRSV DNA vaccine, provide theoretical data for 
clinical application fundamental data and establish foundation for further development of new vaccine adjuvants.

\section{II.MATERIALS AND METHODS}

\section{A. Plasmid, strains, vaccine and animal}

PRRSV-SCQ Isolate ORF5, ORF6 full-length cDNA plasmid pMD19-T-ORF5, pMD19-T-ORF6, E.coli DH5 $\alpha$, Cos-7 cell and Eukaryotic expression vector pCI-neo are kept in self laboratory.pMD19-T simple Vector is production of TaKaRa Biotechnology (Dalian) Co.,Ltd. PRRSV live vaccine is production of Shanghai Hayley Biotechnology Co.,Ltd. Four weeks old mice are purchased from Laboratory Animal Research Center of SiChuan University.

\section{B. Reagent and instrument}

T4 DNA Ligase, various restriction endonuclease were purchased from TaKaRa Biotechnology, Dalian, China. Protein marker was purchased from TaKaRa Biotechnology, Dalian, PR China. FuGENE® HD Transfection Reagent, Endo-Free Plasmid Kit I, were purchased from Omega Biotech, Shanghai,China. IFN$\gamma /$ IL-4 ELISA detection Kit of Mouse were purchased from Excel Biotech, Shanghai, China. Inverted fluorescence microscope was purchased from Nikon Instruments, Shanghai, China.

\section{Primers devise and PCR augment}

According to PRRSV SCQ gene array in Gene Bank stub (landing NO: 379479), devised four primers.6RA and 6RB have the common 6F (table 1), 5R and $5 \mathrm{~F}$ expend complete genes for 630bp; 6RA and 6F expend ORF6 genes for 537bp (contain ending codon); $6 \mathrm{RB}$ and $6 \mathrm{~F}$ expend ORF6 genes for 546bp (no ending condon); 7R and7F expend Hsp70 genes for 1884bp.All the primers were compounded by invitrogen company.

Table 1. Sequences of PCR primers

\begin{tabular}{cc}
\hline primer & Sequences $\left(5^{\prime} \rightarrow 3^{\prime}\right)$ \\
\hline $5 \mathrm{~F}$ & GAATTCATGTTGGAGAAATGCTTGACCGCGGGCTGTTGCTC \\
$5 \mathrm{R}$ & TCTAGACTCGAGGGATCCAGAGCCAGAACC \\
& AGACGACCCCATTGTTCCGC \\
$6 \mathrm{~F}$ & GGATCCGGTTCTGGCTCAGGTTCTATGGGGTCGTCCTTAGATGAC \\
$6 \mathrm{RA}$ & GGCTCATCTAGATTATTTGGCATATTTGACAAGGTTTACCACTCC \\
$6 \mathrm{RB}$ & GGATCCGGTTCTGGCTCAGGTTCTATGGGGTCGTCCTTAGATGAC \\
$7 \mathrm{~F}$ & TCTAGAGGTTCTGGCTCAATGGCTCGTGCGGTCGGGATCGACCTCGGG \\
$7 \mathrm{R}$ & CCCGGGTCACTTGGCCTCCCGGCCGTCGTCGACCACCTCCGCGTCCAC \\
\hline
\end{tabular}

Label: the lineation is enzyme location:

GAATTCA(EcoR I), GGATCC(BamHI), TCTAGA(XbaI), CCCGGG(SmaI)

\section{Construction of pCI-ORF5-ORF6 $A$ and pCI-ORF5-ORF6B-Hsp 70}

The tandem gene T-ORF5-ORF6 was obtained by combining the Gene Fragments ORF5 and ORF6. The fragment was retrieved by double-enzyme digestion by EcoRI/XbaI and then cloned into pCI-neo vector to obtain the eukaryotic recombinant expression plasmids containing pCI-ORF5-ORF6A (with terminator) and pCI-ORF5-ORF6B (without terminator). Then the plasmids were identified by EcoR I /XbaI. After enzyme retrieval Hsp70 with $\mathrm{XbaI} / \mathrm{SmaI}$ and fusion with ORF5-ORF6B, Eukaryotic expression plasmid pCI-ORF5-ORF6B-Hsp70 is constructed.

Ethel expression of recombinant plasmid in COS-7 cell

\section{a. Transfection}

pCI-ORF5-ORF6A and pCI-ORF5-ORF6B-Hsp70 Expression plasmid DNA were extracted using Endo-Free Plasmid Kit I. Cos-7 cells were grown to $70 \%$ in a six-well plate and then transfected with the expression vectors using FuGENE® HD Transfection Reagent, as specified by the manufacturer. at the same time, bank and pCI-neo are the contrast groups.

\section{b. Western-blotting analysis}

The collected precipitation was washed twice with a phosphate buffer solution (PBS), lysed in lysis buffer, and boiled for 10 minutes at $72 \mathrm{~h}$ after transfection. The supernatant after centrifugation were assayed by sodium dodecy sulfate-polyacrylamide gel electrophoresis (SDS-PAGE) and Western-blotting with porcine-anti PRRSV hyper immune serum as primary antibody and sheep anti-porcine IgG marked with alkaline phosphates as secondary antibody.

\section{F. Animal grouping and immunity}

Five groups of four weeks old female Kunming mice were injected three times, at 2-week intervals, into the femoribus internus muscle with $0.1 \mathrm{mg}$ of plasmid DNA containing pCI-neo, pCI-ORF5-ORF6A, pCI-ORF5-ORF6B-Hsp70, live vaccine and control group. Procaine hydrochloride was injected into the same position 3 days before immunization. The serum was separated with tail tip blood sampling and stored at $-20^{\circ} \mathrm{C}$ every two weeks. At one time, on the day of 
1d,3d,7d,14d,28d,56d,120d after immunity, pick up the tissue core and liver, lung and kidney, muscle and brain ,each group use two mouse. The sample will put to EP tube and stored at $-20^{\circ} \mathrm{C}$.

\section{a.Splenic Lymphproliferation Response(MTT)}

Mouse were killed by cervical dislocation. Spleen was removed by conventional sterile methods, grinded and passed through steel mesh. After placed into the incubator for $2 \mathrm{~h}$, the steel mesh was washed with 8-10mL DMEM (without serum), splenic lymphocytes suspension was collected and centrifuged (1000r/min,10min). Supernatant was removed and precipitation was added $2 \mathrm{~mL}$ erythrocyte lysis buffer ( $\mathrm{NH} 4 \mathrm{Cl}$ ) for $5 \mathrm{~min}$ at RT, and neutralized the erythrocyte lysis buffer with $10 \mathrm{~mL}$ nutritive medium and centrifuged (1000r/min,10min). Splenic lymphocytes concentrations adjusted to $1 \times 10^{7}$ cells $/ \mathrm{ml}$ with DMEM supplemented with $10 \%$ fetal calf serum, and $100 \mu \mathrm{L}$ cells were incubated with concanavalin $\mathrm{A}(100 \mu \mathrm{g} / \mathrm{mL})$ in a 96 -well microplates into the incubator containing $5 \%$ of $\mathrm{CO} 2$ for $60 \mathrm{~h}$ at the temperature of $37^{\circ} \mathrm{C}$, repeated three times. Supernatant was removed and the cells were incubated with $10 \mu \mathrm{L}$ of MTT solution $(5 \mathrm{mg} / \mathrm{mL})$ for another $4 \mathrm{~h}$ at $37^{\circ} \mathrm{C}$, and added $100 \mu \mathrm{L}$ of DMSO for $30 \mathrm{~min}$. The OD value was recorded at the $570 \mathrm{~nm}$ was determined using a microplate reader. The proliferation index was calculated according to the following formula with statistically significant difference.

Proliferation Index $=\left(\mathrm{OD}_{570}\right.$ mean for treatment cell/ $\mathrm{OD}_{570}$ mean for control cell)

\section{b. Detection of Splenic T-Lymphocyte Subsets by Flow Cytometry (FCM)}

Blood samples were collected from the ocular artery directly after eyeball removal and treated with anticoagulant therapy $56 \mathrm{~d}$ after first immune. The samples were mixed $2 \mathrm{ml}$ ammonium chloride in test tubes and stored in the refrigerator at $4^{\circ} \mathrm{C}$ for $10 \mathrm{~min}$.Supernatant was removed after centrifuging at $1500 \mathrm{rpm}$ for $5 \mathrm{~min}$. The cells were collected by washing with $5 \mathrm{ml}$ of PBS twice. The mixture with $0.5 \mathrm{mg} / \mathrm{ml}$ CD3+/APC $2 \mu \mathrm{L}, 0.1 \mathrm{mg} / \mathrm{mL}$ CD4+/PE, CD8+/FITC 10 $\mu \mathrm{L}$ was kept in the refrigerator at $4^{\circ} \mathrm{C}$ for $30 \mathrm{~min}$ and supernatant was discarded after centrifuging as before. The cells at the bottom of the tube were suspended in
$1 \mathrm{ml}$ of PBS and analyzed on flow cytometer while data were analyzed using Cell quest software.

c. IL-4 and IFN- $\gamma$ cytokine ELISA

The IL- 4 and IFN- $\gamma$ cytokine levels in the cell culture supernatants obtained by tail tip blood sampling were measured using PharMingen OptEIA ${ }^{\mathrm{TM}}$ ELISA kits according to the manufacturer's instructions.

d. PCR detection of DNA vaccine distribution in mice Internal organs were taken samples, grinding, freezing and thawing 3 times and taken $400 \mathrm{ul}$ to centrifuge $(12000 \mathrm{~g} / \mathrm{min}, 5 \mathrm{~min})$ at $4^{\circ} \mathrm{C}$. Supernatant was responded with $500 \mu \mathrm{l}$ saturated phenol, and added another $200 \mu \mathrm{l}$ saturated phenol twice. Added 200ul $\mathrm{CHCl} 3$ and resupended supernatant with 2 times the volume of isopropanol and steted for $10 \mathrm{~min}$ at $\mathrm{RT}$, centrifuged(12000r/min,5min)at $4{ }^{\circ} \mathrm{C}$. Removed the supernatant and washed the precipitate and the wall with $75 \%$ of the deep freeze ethanol, centrifuged $(12000 \mathrm{~g} / \mathrm{min}, 5 \mathrm{~min})$ at $4{ }^{\circ} \mathrm{C}$, removed the supernatant again, and dried at room temperature precipitation. Dissolved with 30ul TE containing RNA enzymes for $30 \mathrm{~min}$ at $37^{\circ} \mathrm{C}$, stored the DNA at $4^{\circ} \mathrm{C}$. According described method 1.3, each group were detected by PCR amplification, respectively, taken $5 \mathrm{ul}$ of PCR products and added appropriate amount of bromophenol blue in agarose gel electrophoresis.

\section{RESULTS}

A. Construction and identification of eukaryotic recombinant expression plasmid

Enzyme identification results confirmed that pCI-ORF5-ORF6A, pCI-ORF5-ORF6B-Hsp70 were constructed successfully.(Fig1,2)

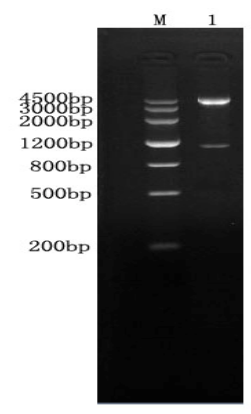

Fig 1 Enzyme digestion profile of pCI-ORF5-ORF6A 
M:Marker III ; 1:EcoR I /XbaI enzyme identification of plasmid pCI-ORF5-ORF6A(1167bp)

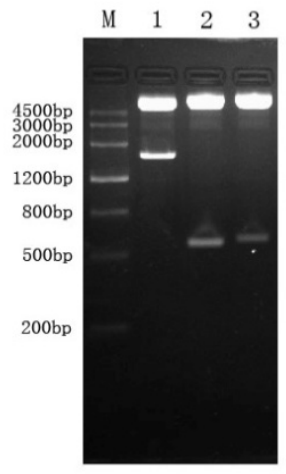

Fig 2 Enzyme digestion profile of pCI-ORF5-ORF6B-Hsp70

M:MarkerIII; 1:Hsp70 plasmid digestion by EcoRI/BamHI(1884bp); 2:ORF6B plasmid digestion by BamHI/XbaI(546bp); 3: ORF5 plasmid digestion by $X b a \mathrm{I} / \operatorname{SmaI}(630 \mathrm{bp})$

B. Expression of recombinant plasmid in Cos-7 cells a. Western-blotting analysis

The cells transfected with pCI-ORF5-ORF6A and pCI-ORF5-ORF6B-Hsp70 were cracked and tested by SDS-PAGE. The blot was then reacted with porcine anti-PRRSV hyper-immune serum. Two reaction bands were observed at $42.7 \mathrm{Ku}$ and $112.2 \mathrm{Ku}$ and the sizes were consistent with expectation, which proved the successful expression of the constructed recombinant plasmid (Fig 3).

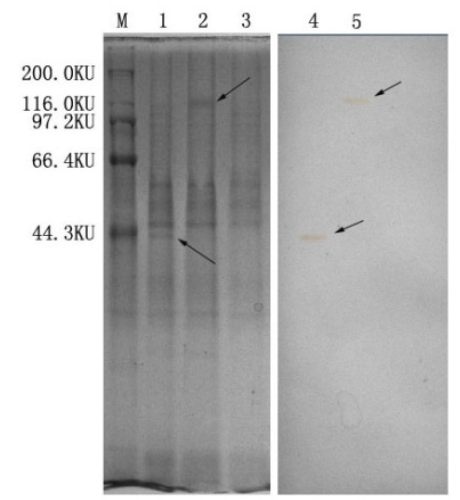

Fig3Expression of PCI-ORF5-ORF6B-Hsp70 protein in COS-7cells detected by Western-blotting

M:high molecular weight protein marker; 1:Express result of PCI-ORF5-ORF6A; 2:Express result of pCI-ORF5-ORF6B-Hsp70; 3:pCI-neo as the negatine control; 4:pCI-ORF5-ORF6A result of Western-blotting; 5:pCI-ORF5-ORF6B-Hsp70 result of Western-blotting.
In Table 1, before immunization, the propagation ability of each group was not different significantly (P> 0.05); After the first immunization, the OD values of each group in $14 \mathrm{~d}$ were increased, especially the pCI-ORF5-ORF6A and pCI-ORF5-ORF6B-Hsp70 were significantly higher than the comparison groups. So, both pCI-ORF5-ORF6A and pCI-ORF5-ORF6B-Hsp70 can stimulate lymphocyte propagation, but no significant difference between them.After the second immunization, the propagation ability of both 14d pCI-ORF5-ORF6A and pCI-ORF5-ORF6B-Hsp70 groups was significantly different from the comparison groups, and the OD value of the pCI-ORF5-ORF6B-Hsp70 group was higher than the pCI- ORF5-ORF6A group, but the difference was not significant. After the third immunization, the propagation ability of the $14 \mathrm{~d}$ pCI-ORF5-ORF6B-Hsp70 group was significantly different from its comparison and pCI-ORF5-ORF6A,so,it proves that Hsp70 as adjuvants which can stimulate lymphocyte propagation effectually. (Table1)

\section{c. Detection of Splenic T-Lymphocyte Subsets}

T Lymphocyte Subsets in Peripheral Blood was taken at 56 days after primary immunization and 15000 cells were measured with a flow cytometer. Statistical analysis mean data are given by $\mathrm{X} \pm \mathrm{SD}$ and the differences between paired data were analyzed using paired t-test. A significant increase $(p<0.05)$ in the absolute numbers of both $\mathrm{CD}^{+}$and $\mathrm{CD} 8^{+} \mathrm{T}$ cells in groups immunized with pCI-ORF5-ORF6A and pCI-ORF5-ORF6B-Hsp70 compared with that with empty plasmid (pCI-neo) and PBS. (Table2).

\section{d. IL-4 and IFN- $\gamma$ cytokine ELISA}

After primary immunization, the IL- 4 and IFN- $\gamma$ cytokine levels in the cell culture supernatants obtained by tail tip blood sampling every 14 days were measured using PharMingen OptEIA ${ }^{\text {TM }}$ ELISA kits. IL-4 and IFN- $\gamma$ cytokine levels of all immunized groups were increased obviously while group immunized with pCI-ORF5-ORF6B-Hsp70 showed much higher level than that immunized with pCI-ORF5-ORF6A $(\mathrm{P}<0.01)$.Results confirmed that Hsp70 as an adjuvant

\section{b. Splenocytes propagation (MTT)}


can significantly enhance the ability of Splenic Lymphocyte in Peripheral Blood to induce IL-4 and IFN- $\gamma($ Fig 4,5).

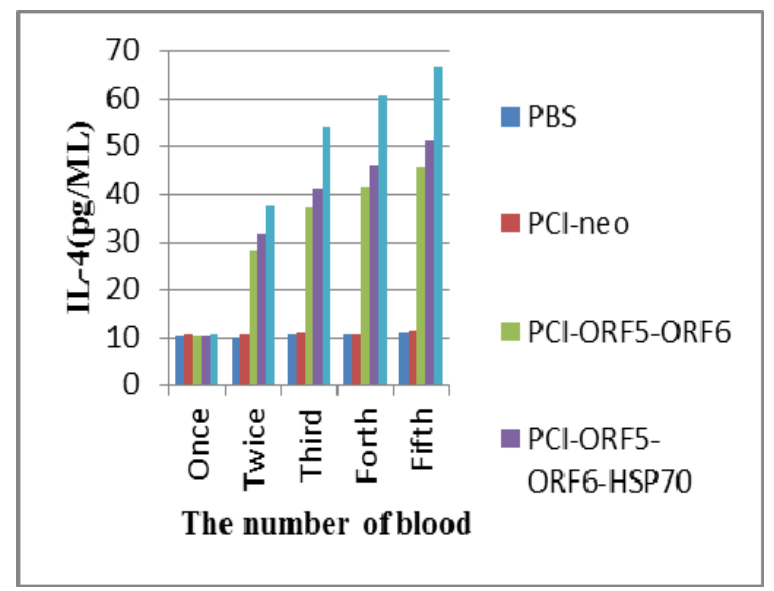

Fig.4 Level of IL-4 in blood of mice

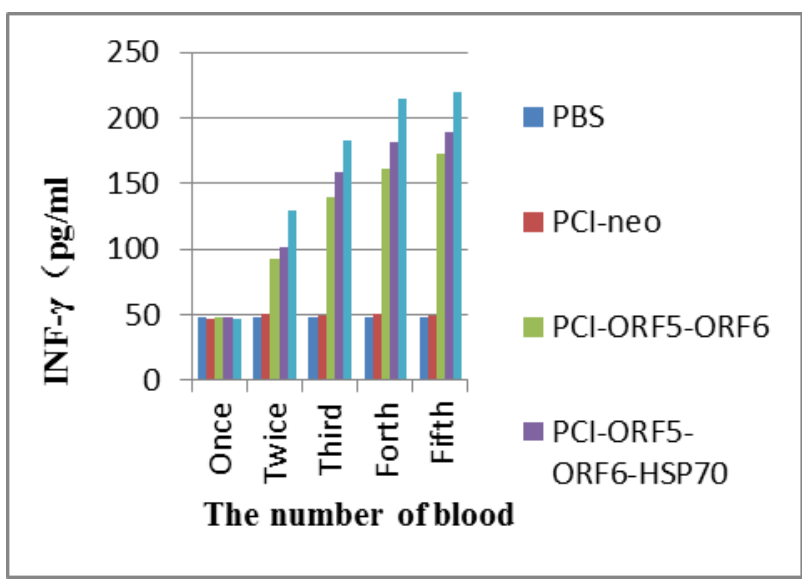

Fig.5 Level of IFN- $\gamma$ i n blood of mice

Table1 OD value of proliferation of mice speen lymph cells in different groups

\begin{tabular}{|c|c|c|c|c|}
\hline Groups & Before immunization & 14d after once & 14d after twice & $14 d$ after third \\
\hline PBS & $0.204 \pm 0.09$ & $0.209 \pm 0.08$ & $0.213 \pm 0.11$ & $0.220 \pm 0.12$ \\
\hline pCI-neo & $0.202 \pm 0.07$ & $0.253 \pm 0.09$ & $0.298 \pm 0.14$ & $0.304 \pm 0.05$ \\
\hline pCI-ORF5-ORF6A & $0.215 \pm 0.05$ & $0.404 \pm 0.03$ & $0.436 \pm 0.10$ & $0.486 \pm 0.02$ \\
\hline pCI-ORF5-ORF6B-Hsp70 & $0.219 \pm 0.11$ & $0.411 \pm 0.08$ & $0.452 \pm 0.07$ & $0.513 \pm 0.11$ \\
\hline $\mathrm{CH}-1 \mathrm{R}$ live vaccine & $0.228 \pm 0.06$ & $0.466 \pm 0.06$ & $0.509 \pm 0.10$ & $0.596 \pm 0.13$ \\
\hline
\end{tabular}

Table 2The numbers of mice spleen T lymphocyte subgroups indifferent groups

\begin{tabular}{|c|c|c|c|}
\hline Groups & $\mathrm{CD}^{+}$ & $\mathrm{CD8}^{+}$ & $\mathrm{CD4}^{+} / \mathrm{CD8}^{+}$ \\
\hline PBS & $37.97 \pm 1.64$ & $17.98 \pm 1.59$ & $1.62 \pm 0.55$ \\
\hline pCI-neo & $39.17 \pm 1.93$ & $18.75 \pm 1.12$ & $1.63 \pm 0.82$ \\
\hline pCI-ORF5-ORF6A & $46.86 \pm 1.57$ & $21.29 \pm 1.59$ & $1.81 \pm 1.02$ \\
\hline pCI-ORF5-ORF6B-Hsp70 & $53.37 \pm 1.83$ & $21.70 \pm 2.14$ & $2.12 \pm 1.78$ \\
\hline $\mathrm{CH}-1 \mathrm{R}$ live vaccine & $54.33 \pm 2.38$ & $24.26 \pm 1.72$ & $2.08 \pm 1.23$ \\
\hline
\end{tabular}

\section{e. PCR detection of DNA vaccine distribution in mice}

In the sample, on $28 \mathrm{~d}$ after immunity, pCI-ORF5-ORF6A be checked out positive firstly in the heart and muscle tissue, on 56d, muscle and brain be checked out positive, on $120 \mathrm{~d}$, all of the tissue be checked out negative; pCI-ORF5-ORF6B-Hsp70 be checked out positive before $14 \mathrm{~d}$, on $28 \mathrm{~d}$,brain be checked out negative firstly, on $120 \mathrm{~d}$, all of the tissue be checked out negative but muscle; $\mathrm{CH}-1 \mathrm{R}$ live vaccine be checked out positive before $14 \mathrm{~d}$, on $56 \mathrm{~d}$, brain be checked out negative firstly, on $120 \mathrm{~d}$,all of the tissue be checked out negative but muscle; PBS and pCI-neo are the control group, and be checked out negative.

\section{DISCUSSION}

Whether the DNA vaccine construction is succeed depends on the exogenous gene immunogenicity intensity and express the discretion of in vitro activity[7]. Therefore, In constructing the DNA vaccine, restructured plasmid will be test in vitro firstly and checkt its expression activity, for the DNA vaccine inside animals success express support. For this purpose, the scholars have conducted the thorough research to PRRSV, and found that the most appropriate exogenous gene by the 
time. They are and $\mathrm{N}$ protein (encode by ORF7 gene), Have the virus protein structure characteristics. Duran[8] let this gene transfection cell and obtain expression, and ascertain ORF5 is one of the selection genes. PING-AN[9] let E /M protein transfection cell and obtain expression together, also indentify that not only ORF5/ORF6 genes may by way of the PRRSV DNA vaccine gene feasibility, but also expresses altogether can have the synergism action and enhancement immunity effect. From that point, we cloned the two genes ORF5/ORF6, and Construction of Eukaryotic recombinant expression vector pCI-ORF5-ORF6A. Mice immunized result showed that DNA vaccine pCI-ORF5-ORF6A be able to induce mice production humoral immunity and cellular immunity effectively. The number of $\mathrm{CD} 3+, \mathrm{CD} 4+, \mathrm{CD} 8+$ T-Lymphocyte subsets all increased. The result showed that the Eukaryotic recombinant Expression Vector of pCI-ORF5-ORF6A will espress and cause immune response.

Current, there is a lot of methods to increased the antibody levels and the effect of NK cells of DNA vaccine, the expression of multiple antigen thread, codon optimization and adjuvant used[10]. As a stress protein, HSP70 is highly conserved in evolution. Except for chaperone features, cell protection, antiapopotic features, antioxidant features, there is also a important features, immune function[11-13]. The research showed that HSP70 can protect the physiological function by inhibiting IL-1 and interferon on the induction of NO synthase. Mehlen.P found that the content of glutathione would increase by the stimulation of HSP, and the glutathione will protect cells from tnf and other cytokines attack[14]. Another study showed that HSP70 can be used as adjuvants enhance the immune effect of peptide or polysaccharide, Peptides and mtHSP70 crosslinked will educe adjuvant efeffective charge of defect. The carboxyl terminal of HSP70 was used as an adjuvant enhancing immunity effectiveness of the fusion gene vaccine HCA661[15]. Das found that the epitope immune effect of JEV will be enhanced by confluence the epitope and HSP70[16].

Continuous existence of DNA vaccine in animal body meant continuous expression of gene vaccine, and quickly distribution of vaccine would be helpful for the lasting and effective expressing in different tissue[17]. Dou[18] detected the recombinant plasmid after immunizing $\mathrm{BALB} / \mathrm{c}$ mouse by both gene gun bombardment and intramuscular injection with recombinant plasmid, and found that the lasting time of DNA in mouse BALB/c muscle is dependent on the dose of use. In this research, we injected DNA vaccine into the mouse muscle, then pCI-ORF5-ORF6A and pCI-ORF5-ORF6B-HSP70 were detected in the immunization muscle of heart, liver, spleen and lung and in the brain from day 1 after injection to day 120 . The results meant DNA vaccine could exist relatively long in muscle cells, so the target gene could continuously express in the tissue and immunize effectively. In this study, the immunological results showed that the immune effect of fusion expression plasmid pCI-ORF5-ORF6B-HSP70 is better than the plasmid pCI-ORF5-ORF6A. No matter the cellular immunity nor the humoral immunity was effectively enhanced. This also showed that the HSP70 can enhance the immune effect of DNA vaccines and HSP70 can be used as an adjuvant.

\section{ACKNOWLEDGMENT}

The Study Supported by Program for Changjiang Scholars and Innovative Research Team in University (PCSIRTU, PROJECT No. IRT0848).

\section{REFERENCES}

[1] WEnSVOORT G, TERPSTRA C, POL J, et al. Mystery swine disease in The Netherlands: the isolation of Lelystad virus [J]. The Veterinary Quarterly, 1991, 13(3): 121.

[2] SHI-WEN T. Advances on Highly Pathogenic Porcine Reproductive and Respiratory Syndrome (PRRS)[J] [J]. Guizhou Agricultural Sciences, 2009, 6 [3] JIANG Y, FANG L, XIAO S, et al. A suicidal DNA vaccine co-expressing two major membrane-associated proteins of porcine reproductive and respiratory syndrome virus antigens induce protective responses [J]. Biotechnology letters, 2009, 31(4): 509-18.

[4] ZHOU Y, HAO X, TIAN Z, et al. Highly virulent porcine reproductive and respiratory syndrome virus emerged in China $[\mathrm{J}]$. Transboundary and Emerging Diseases, 2008, 55(3 - 4): 152-64. 
[5] ZHOU Y, YU H, TIAN Z, et al. Monoclonal antibodies and conserved antigenic epitopes in the $\mathrm{C}$ terminus of GP5 protein of the North American type porcine reproductive and respiratory syndrome virus $[\mathrm{J}]$. Veterinary microbiology, 2009, 138(1-2): 1-10.

[6] VOS M, HAGEMAN J, CARRA S, et al. Structural and Functional Diversities between Members of the Human HSPB, HSPH, HSPA, and DNAJ Chaperone Families [J]. Biochemistry, 2008, 47(27): 7001-11.

[7] SELVIN P, HA T. Single-molecule techniques: a laboratory manual [M]. Cold Spring Harbor Laboratory Pr, 2008.

[8] DURAN J, CLIMENT I, SARCASECA J. Baculovirus Expression of Porcine Reproductive and Respiratory Syndrome Virus Strain Olot/91: Involvement of ORF3 and ORF5 proteins in protection [J]. Virus genes, 1997, 14(19-29).

[9] PING-AN X, ZHAN-GUO D, BIN Z, et al. Pseudotyping of Murine Leukemia Virus Particles with Porcine Reproductive and Respiratory Syndrome Virus M Protein-mediated E Protein [J] [J]. Chinese Journal of Virology, 2008, 5.

[10] DAUGAARD M, ROHDE M, J TTEL M. The heat shock protein 70 family: Highly homologous proteins with overlapping and distinct functions $[\mathrm{J}]$. FEBS letters, 2007, 581(19): 3702-10.

[11] TSAN M, GAO B. Heat shock proteins and immune system [J]. Journal of leukocyte biology, 2009, 85(6): 905.

[12] ZHANG K, ZHAO T, HUANG X, et al. Preinduction of HSP70 promotes hypoxic tolerance and facilitates acclimatization to acute hypobaric hypoxia in mouse brain [J]. Cell Stress and Chaperones, 2009, 14(4): 407-15.

[13] KOKOLAKIS G, KRITSIDIMA M, TKACHENKO T, et al. Two hsp23 genes in the Mediterranean fruit fly, Ceratitis capitata: structural characterization, heat shock regulation and developmental expression [J]. Insect Molecular Biology, 2009, 18(2): 171-81.

[14] SINGH M, REDDY M, MATHUR N, et al. Induction of hsp70, hsp60, hsp83 and hsp26 and oxidative stress markers in benzene, toluene and xylene exposed Drosophila melanogaster: Role of ROS generation $[\mathrm{J}]$. Toxicology and applied pharmacology, 2009, 235(2): 226-43.

[15] VAN DEN P, WOLLMANN G. COMPOSITIONS AND METHODS OF USE OF AN ONCOLYTIC VESICULAR STOMATITIS VIRUS [M]. US Patent App. 20,100/172,877.

[16] DAS S, LAXMINARAYANA S, CHANDRA N, et al. Heat shock protein 70 on Neuro2a cells is a putative receptor for Japanese encephalitis virus [J]. Virology, 2009, 385(1): 47-57.

[17] AN CHUN C, MING SHU W,NI GEN X,et al. Construction of ORF5 gene vaccine against PRRSV and detection of its safety and immunogenicity $[\mathrm{J}]$. Chinese Journal of Veterinary Science and Technology,2004,35(1):27-35.

[18] WEN BO D,AN CHUN C, MING SHU W,et al .PCR Detects the PRRSV of ORF5 Gene Vaccine in BALB/c Mice Vaccinated via Gene Gun Bombing[C]. 2005.

ZUO LAN(1987-), Student of master degree, major is molecular biology of Animal infectious diseases. Key Laboratory of Animal Disease and Human Health of Sichuan Province \& Animal Biotechnology Center, College of Veterinary Medicine of Sichuan Agricultural University, 46\# Xinkang Road, Yucheng district, Ya'an 625014, Sichuan province of China. Tel.: +86 835 2882423; E-mail address:joanna_0408@yahoo.com.cn.

YAN Qi-gui is the corresponding authors. Key Laboratory of Animal Disease and Human Health of Sichuan Province \& Animal Biotechnology Center, College of Veterinary Medicine of Sichuan Agricultural University, 46\# Xinkang Road, Yucheng district, Ya'an 625014, Sichuan province of China. Tel.: +86 835 2882423; E-mail address: yanqigui@126.com. 